\title{
Wettbewerb mit Vorbehalten
}

\section{Vielfalt und Ambivalenz in der neuen Marktkultur des Krankenhauswesens}

\author{
Von Ingo Bode und Markus Märker
}

Zusammenfassung: Marktorientierte Steuerung hat im Wohlfahrtsstaat sowie im Gesundheitswesen zunehmend an Bedeutung gewonnen, ihre Verarbeitung durch die betroffenen Akteure ist bislang aber unterbelichtet geblieben. Am Beispiel des deutschen Krankenhaussektors und im Rekurs auf Bestände der Wirtschaftssoziologie sowie der Strukturationstheorie untersucht dieser Beitrag, wie Wettbewerb bei Kollektivakteuren auf der intermediären Steuerungsebene des Klinikwesens kognitiv prozessiert wird. Empirische Grundlage sind Befunde aus Experteninterviews sowie akteursspezifischen Dokumenten mit Öffentlichkeitsbezug. Die Analyse umfasst Kostenträger, Betreiber, Länderbehörden (als Planer) sowie verschiedene Berufskollektive und führt vor Augen, dass all diese Gruppen eine sehr unterschiedliche Vorstellung von dem haben, was Wettbewerb ist und was er sein soll. Insgesamt zeigt sich innerhalb des fraglichen Akteurskreises ein hohes Maß an Ambivalenz. Dies korrespondiert mit spezifischen Interesseninterpretationen, aber auch mit divergierenden Regulierungskonzepten. Qualitätswettbewerb erweist sich als der größte gemeinsame Nenner. Der diesbezügliche Konsens ist zwar letztlich inkonsistent, aber er scheint maßgeblich zur Stabilisierung der Wettbewerbsidee als solcher beizutragen.

\section{Einleitung}

Die systematische Nutzung des Wettbewerbsprinzips für die Organisation des Wohlfahrtsstaats ist mittlerweile zur Selbstverständlichkeit geworden, auch bei der Gesundheitsversorgung (Nullmeier 2007; Grzeszick 2010). Aus soziologischer Sicht wirft sein Eindringen in gesellschaftliche Felder, in denen es lange Zeit eine marginale Rolle gespielt hat, jedoch grundlegende Fragen auf, nicht zuletzt die nach der kognitiven Verarbeitung dieser Entwicklung durch feldrelevante Akteure als wesentlichem Moment der Prozessierung institutionellen Wandels. Die Beantwortung dieser Frage ist keine reine akademische Übung: Denn die Neuregulierung eines gegebenen gesellschaftlichen Sektors funktioniert - wie nicht zuletzt die verwaltungswissenschaftliche Implementationsforschung lehrt (für viele: Mayntz 2009) - nur dann, wenn sie akteursübergreifend internalisiert wird; ansonsten ist sie permanent störanfällig.

In der Wohlfahrtsstaatsforschung spielen auf diese Prozessdimension institutionellen Wandels bezogene Analysen bislang kaum eine Rolle. Das gilt besonders für die kognitive Verarbeitung dessen, was wir die neue Marktkultur im Gesundheitssystem nennen möchten. Es geht dabei um Kultur im Sinne eines diskursiv vorherrschenden und von wesentlichen institutionellen Akteuren nicht in Frage gestellten Ordnungsmodells, in dem die Tugenden des interorganisationalen Wettbewerbs allgemein als gewinnbringend gelten und die wenigstens partiell marktförmige Steuerung von Versorgungsprozessen als zeitgemäß und innovativ gilt. Das bedeutet nicht, dass alle in das System involvierten Akteure mit den Begriffen Markt und Wettbewerb das Gleiche assoziieren bzw. eine gleichermaßen affirmative Haltung einnehmen. Aber wie (auch) das im Weiteren präsentierte Material zeigt, stellt sich gegenwärtig niemand offen gegen das Ordnungsprinzip als solches.

Ein interessanter Fall ist diesbezüglich das Krankenhauswesen. Die Regulierung dieses Sektors, die Organisationspolitik der Kliniken, die Sprache der federführenden Akteure in 
Politik und Wissenschaft ${ }^{1}$ und auch die Wahrnehmung der beruflich in die Gesundheitsversorgung Involvierten sind seit vielen Jahren auf Markt und Wettbewerb ,gepolt“. Zwar gibt es Debatten über Folgeprobleme (Manzeschke 2010), die auch die Medienöffentlichkeit erreichen - die einschlägigen Stichwörter sind Kliniksterben, Falschabrechnungen und „geschobene" Organtransplantationen. Für die meisten ist der Wettbewerb aber hier nur falsch reguliert und kein Fundamentalproblem - insofern dominiert die Marktkultur.

Allerdings ist unklar, wie das Wettbewerbsprinzip - gewissermaßen unterhalb der Oberfläche dieser Deutungsfolie - im Alltag des Krankenhauswesens ankommt. Immerhin war für die Kultur der Moderne stets grundlegend, dass Gesundheit keine Ware ist, sondern ein gesellschaftlich ,gepflegtes“ Grundrecht. Dabei galten die Asymmetrie der Informationsverteilung oder auch die Bedeutung vertrauensbasierter Ko-Produktion in der Gesundheitsversorgung lange Zeit als Gegenanzeige zu Marktsteuerung. Das Mindeste, was man vermuten kann, ist also, dass deren Einführung in das Gesundheitswesen Zielkonflikte provoziert (Hessinger 2009).

Die Frage, wie Akteure des Feldes mit der neuen Marktkultur umgehen, berührt nicht nur die Berufstätigen vor Ort. Vielmehr wird die Kultur des Feldes - zumal in Deutschland maßgeblich durch organisationsübergreifende Instanzen ,gelebt“: Dazu zählen politische Institutionen (Regierung, Parteien), aber auch Fachapparate der Ministerien (v.a. der Länder), diverse administrative Körperschaften sowie Zusammenschlüsse von Trägern und Berufsgruppen. Diese intermediären Akteure, die die „operationale Governance“ (Hill / Hupe 2009) des Feldes (mit)gestalten, verdichten im Dialog mit ihren Bezugsgruppen kursierende Deutungen und übersetzen sie in strukturbildendes "Steuerungsdenken“. Das macht sie für die Analyse der kognitiven Verarbeitung der neuen Marktkultur besonders interessant.

Kognitive Deutungen sind wesentliche Elemente der Strukturation institutionellen Wandels (im Sinne von Giddens 1992): Der Strukturationsbegriff verweist darauf, dass Veränderungen sozialer Zustände in einem Zusammenspiel von institutionalisierten Regeln und akteursseitigen „Enaktierungen“ vollzogen werden. Die kognitive Seite dieses Prozesses ist also von zentraler Bedeutung, wenn es um die Implementierung neuer Steuerungen geht. Nachfolgend wird argumentiert, dass die neuen Wettbewerbsverhältnisse sehr vielfältig gedeutet werden, was auf ,marktkulturelle“ Ambivalenz hindeutet. Die Deutungsvielfalt korrespondiert teilweise mit je spezifischen Interesseninterpretationen, doch findet sich Ambivalenz auch jenseits davon. Paradoxerweise spricht vieles dafür, dass sie die Verankerung des Wettbewerbsprinzips befördert.

Wir entwickeln dieses Argument im Rückgriff auf erste Befunde eines mit der Governance des heutigen Krankenhauswesens befassten Forschungsvorhabens. ${ }^{2}$ Am Anfang steht eine theoretische Betrachtung, die - v.a. im Rekurs auf wirtschaftssoziologische und strukturationstheoretische Überlegungen - Wettbewerb als uneindeutiges Ordnungsprinzip fasst und die kritische Bedeutung ,kognitiver Praxis“ im Umgang mit konkurrierenden institutionellen Logiken beleuchtet. Anschließend geht es um die Marktordnung im deutschen Krankenhauswesen, besonders darum, wie das Wettbewerbsprinzip hier konkret zur Geltung kommt. So kann dann im dritten Abschnitt - durch die Entfaltung von v.a. aus Experteninterviews gewonnenen synthetisierten Argumentationsfiguren - analysiert werden, wie bei intermediären Akteuren des Feldes über dieses Prinzip reflektiert wird und welche Zonen der Ambivalenz dabei zum Vorschein kommen. Den Abschluss bildet eine theoretische Zuspitzung der Befunde.

1 Begriffe wie „Managed Care“ und Gesundheitsmarkt gehören heute zum Standardvokabular dieser Akteure (Amelung 2009; Rau et al. 2009).

2 Es handelt sich um das von der DFG bis 2015 geförderte Projekt „Ambivalenzbewältigung im Krankenhauswesen“, das sowohl die Organisationsebene wie auch den hier analysierten Bereich intermediärer Steuerung untersucht. 


\section{Wettbewerb (im Wohlfahrtsstaat) als uneindeutiges und raumschaffendes Ordnungsprinzip}

In den Sozialwissenschaften ist Wettbewerb kein großes Thema. Wie Nullmeier (2000: 192ff) in seiner Gesamtschau zur "Soziologie der Konkurrenz" feststellt, finden sich lediglich in Norbert Elias' Zivilisationstheorie sowie bei Max Weber grundsätzlichere Überlegungen zur sozialen Natur des Wettbewerbs. ${ }^{3}$ Für die Wirtschaftswissenschaften bildet Wettbewerbstheorie hingegen eine eigene Teildisziplin (Nullmeier 2000: 203ff): Hier ist die lange vorherrschende Annahme vollkommener Konkurrenz mittlerweile Modellen gewichen, in denen dynamische Ungleichgewichte die ergebnisoffene Interaktion zwischen Marktteilnehmern sowie die Möglichkeit strategischen Machthandelns Wettbewerbsverhältnisse je markt- bzw. produktspezifisch ausgestalten (Olten 1998). Man kann (bereits) daraus schließen, dass im (Markt-)Wirtschaftssystem grundsätzlich Vielfalt und Offenheit bezüglich möglicher Konkurrenzsituationen bestehen. Dabei ist zu beachten, dass gewöhnliche Märkte (etwa für Konsumgüter) hochgradig institutionell reguliert sein können (z.B. in puncto Verbraucher- und Umweltschutz) - die Existenz ordnungspolitisch begründeter Regelsysteme ändert nichts daran, dass formal stark konkurrenzorientiert gehandelt wird. ${ }^{4}$

Dies deckt sich mit dem Destillat der neuen Wirtschaftssoziologie, die die soziale Konstruktion von Märkten analysiert, also etwa die „Herausbildung eines positionalen Systems durch Wettbewerb“ (Fuhse / Mützel 2010: 209) oder Prozesse der Marktgenese im Rahmen einer „systematischen Verbindung von Ökonomie, politischem Gemeinwesen und gesellschaftlichem Wertesystem“ (Beckert 1997: 237; s. auch Fligstein 2011). Marktwettbewerb erscheint hier als durch je spezifische marktexterne Institutionen strukturiert und in eine sozial konstruierte Marktkultur eingebettet. Diesbezüglich finden sich zahlreiche Varianten, entsprechend ist potenziell auch ,die Rede vom Wettbewerb [...] mehrdeutig“ (Nullmeier 2000: 236).

Begreift man strukturationstheoretisch (mit Giddens 1992) Ordnungsbildung als Ergebnis eines dialektischen Zusammenspiels von Struktur und Handlung, bei dem Regeln (also auch wettbewerbsbezogene Normen) von Akteuren interpretiert, angewendet und in praxi restrukturiert werden, so ist der eigensinnige kognitive Umgang mit Regeln Kernelement dessen, was man als Strukturation institutionellen Wandels bezeichnen kann. Den durch existierende Regel(strukture)n vorgeprägten Akteuren eröffnet sich ein Raum, innerhalb dessen sie eine „kognitive Praxis“ entfalten, welche wiederum grundlegend ist für „harte“ Gestaltungsprozesse. Mit dem Organisationstheoretiker Weick kann man dabei den konstitutiven Zusammenhang von subjektiven Interpretationen und materieller Gestaltungspraxis als „Enaktierung“ (Weick 1979: 64) begreifen. ${ }^{5}$ Diese vollzieht sich - wie neuere Varianten des soziologischen Neo-Institutionalismus implizieren (vgl. Thornton et al. 2012) - auch organisationsübergreifend: Gibt es Impulse zur Bildung neuer Regelstrukturen, entsteht ein Korridor operationaler Governance, in dem kollektive Akteure institutionelle Logiken kreativ ver- und bearbeiten.

3 Dabei wird dieser bei Elias als Sphäre der Verlustangst begriffen, während Weber ihn mit preisorientierten Tauschbeziehungen im Kapitalismus in Verbindung bringt. Zu nennen sind noch die Arbeiten Pierre Bourdieus zu Machtkonkurrenz und sozialer Distinktion, soweit sie sich auf ökonomischen Wettbewerb beziehen (Fley 2006).

4 Insofern laufen immer wieder anzutreffende Hinweise, das Gesundheitswesen sei in seiner heutigen Verfassung kein Markt (s. etwa Bruckenberger 2006), sozialtheoretisch ins Leere.

5 Dies korrespondiert in der betrieblichen Praxis (die in diesem Beitrag nicht weiter untersucht wird) mit kreativen Anwendungen entsprechender Interpretationsfolien im Kontext organisationaler Indifferenzzonen. 
Genau dies ist der Fall bei der Schaffung von Wettbewerb in staatlich regulierten „Wohlfahrtsmärkten“ (Bode 2005), auf denen Leistungsersteller (z.B. Pflegestationen, Fortbildungseinrichtungen, Kinderheime u.a.) um Aufträge von öffentlichen Kostenträgern oder Transferleistungsbeziehern konkurrieren. ${ }^{6}$ Veränderte Rahmenvorgaben (z.B. Konkurrenzregeln, Vertragsbestimmungen etc.) begründen neue Normalitätserwartungen, wobei es erst im Zuge einer komplikationsträchtigen Reorientierung aller Beteiligten zur Bildung neuer Ordnungsstrukturen kommt. In der entsprechenden kognitiven Praxis werden verschiedene ,institutionelle Logiken" in Anspruch genommen - im Fall des Gesundheitswesens etwa solche, die das Leitbild der „business-like health care“ (Reay / Hinings 2009: 630) stark machen und solche, die vor dem Wettbewerbsimpuls eingeübt worden sind, konkret etwa die Vorstellung universeller Teilhabe- und Versorgungsrechte, wie sie westliche Wohlfahrtsstaaten im Verlaufe des 20. Jahrhunderts ausgebildet haben und auch von maßgeblichen Professionen als „Bedarfsprinzip“ verinnerlicht worden sind. Bedarfs- und Wettbewerbsprinzip verkörpern insofern ,,competing institutional logics“ (Reay / Hinings 2009), die gleichsam als Deutungsfolien in die kognitive Praxis der Akteure einfließen.

Die Vermarktlichung bislang nicht-marktförmig organisierter Sektoren führt zu einer Steigerung von Diversität (Slater / Tonkiss 2001; Neckel 2008), aber zugleich, wie oben bereits erwähnt, zu potenziellen Zielkonflikten (Hessinger 2009; Bär 2011). So veranlassen Finanzierungsformen im Sozial- und Gesundheitswesen, bei denen Einzelleistungen pauschal vergütet werden, dazu, Fallkosten und Fallerträge gegeneinander abzuwägen - bei medizinischen Behandlungen etwa bezüglich des Aufwands für Diagnostik, Abklärung und kollegiale Fallberatung. Nicht wenig spricht dafür, dass sich die involvierten Akteure hier einer permanenten Ambivalenz gegenübersehen - durchaus in Entsprechung zum individuellen Subjekt der Spätmoderne, das innerhalb verschiedener Lebenskontexte konkurrierende „modernitätstypische“ Werte untereinander abwägen muss (Junge 2000). ${ }^{7}$ Wie damit konkret umgegangen wird, bleibt eine empirisch zu klärende Frage.

\section{Die heutige Wettbewerbsordnung im deutschen Krankenhauswesen}

Der deutsche Krankenhaussektor stellt sich heute als eine „mixed economy“ dar, die zwar hochgradig sozialpolitisch gesteuert ist, in der aber die einzelnen Kliniken über wachsende ökonomische Bewegungsspielräume verfügen und eine marktorientierte professionelle Bürokratie $^{8}$ verkörpern. Die Hintergründe dafür sind:

- grundlegende Umstellungen beim institutionellen Modus der Ressourcenausstattung der Kliniken durch die Einführung eines Fallpauschalensystems ${ }^{9}$ in Verbindung mit verstärkter formaler Prozesskontrolle, ${ }^{10} \mathrm{im}$ Rahmen einer überbetrieblichen, (weiterhin)

6 Dadurch sollen öffentlich verantwortete Versorgungsleistungen kosteneffizient(er) erbracht werden. Das impliziert eine Kombination von sozialisierter Nachfrage und konkurrentiellem Angebot einerseits, die Verlagerung wirtschaftlicher Risiken auf Leistungsersteller andererseits. Ein Mix aus „Kundensouveränität“" und intensiverer Qualitätskontrolle soll für Marktkontrolle sorgen.

7 Die entsprechende Gesellschaftsdiagnose geht z.B. davon aus, dass das (spät-)moderne Individuum ständig vor der schwierigen Wahl steht, Individualität und Flexibilität (im Sinne von Anpassungsfähigkeit) miteinander in Einklang zu bringen.

8 Der Begriff stammt von Litwak (1978); die Marktorientierung kommt hinzu. Zur Regulierung des Krankenhaussektors vgl. allgemein Bruckenberger et al. (2006).

9 Die Gesetzliche Krankenversicherung vergütet Kliniken heute nicht mehr real entstandene Behandlungskosten, sondern zahlt Pauschalpreise, die sich am diagnostizierten Krankheitsbild (gelistet in sogenannten „diagnosis-related groups“, DRGs) orientieren. Die einzelnen Kliniken verhandeln mit Kostenträgern Mengenpakete (,case mixes“), wobei staatliche Vorgaben die Steigerung ihrer Gesamtbudgets begrenzen.

10 U.a. durch das Fallpauschalensystem rahmende, „Sicherheitsvorkehrungen“ (Respektierung von Mindestmengen, Höchst- und Mindestliegezeiten, Kodierregeln etc.). 
von intermediären Akteuren ausgestalteten kollektivvertraglichen Feldsteuerung (Strukturplanung, Qualitätssicherung, Einzelbudgetverhandlungen) und in Verbindung mit einer Investitionskostenfinanzierung (durch die Länder), die zunehmend einem dem Fallpauschalenmodell nachempfundenen ,leistungsorientierten“ Förderungsansatz folgt;

- Ansätze eines Angebots- und (partiellen) Preiswettbewerbs, einerseits in Gestalt von Mengenausweitungsstrategien der Kliniken (Spezialisierungen, Einweisermarketing etc.), andererseits im Rahmen sogenannter Integrierter Versorgungsprogramme, die in „Selektivverträge“ gegossen werden und Kliniken veranlassen, eine bestimmte Menge von Behandlungen an Krankenkassen zu ,verkaufen“, um sich damit feste Patientenströme zu sichern - all dies mit der Folge stärker streuender Betriebsergebnisse (Augurzky et al. 2010);

- mehr oder weniger flächendeckend vollzogene organisationsstrukturelle Veränderungen in Gestalt der Einrichtung von Profit-Centern, kennzahlenbasiertem Controlling, ergebnisabhängigen Vergütungen für Führungskräfte, standardisierten Behandlungspfaden (Pfaff / Scheibler 2006; Bär 2011) ${ }^{11}$ sowie von Verschiebungen in der Personalstruktur (mehr Ärzte, weniger nicht-medizinisches Personal, neue Berufsgruppen; Simon 2008), bei gleichzeitig stärker taylorisierter Arbeitsorganisation ${ }^{12}$ und wachsenden Erwartungen an den Ausweis von (formaler) Prozess- und Ergebnisqualität.

In Analysen zu Wirkungen dieser neuen Rahmenordnung wird oft unterstellt, dass sich Kosten der durch sie erzwungenen Umstellungen nach außen (z.B. auf Zulieferer) verlagern lassen oder ein verändertes Management gleiche Versorgungsoutputs mit relativ geringem Ressourceninput (v.a. Personal) gewährleisten kann. Die wenigen verfügbaren Evaluationen bemühen sich um empirische Indizien zur Bestätigung dieser Annahmen (Augurzky et al. 2009). Wieweit nun aber Spannungen, die der Wettbewerbsdruck erzeugt, feldweit durch ,geniale“Steuerungen betriebswirtschaftlich-organisatorischer Art gelöst werden können, ist ungeklärt. In der soziologisch orientierten Folgenabschätzung wird auf Anpassungsprobleme verwiesen, die innerhalb des Sektors anfallen, konkret: Konsequenzen auf der Ebene des medizinischen und pflegerischen Handelns (Braun et al. 2010), welche perspektivisch auch den Versorgungsoutput verändern könnten. Unabhängig davon sind - angesichts der Überlegungen des zweiten Abschnitts - sowohl auf der betrieblichen als auch auf der überbetrieblichen Steuerungsebene Zielkonflikte nicht unwahrscheinlich.

\section{Empirische Befunde auf der intermediären Ebene: Deutungen kollektiver Feldakteure}

In sozialwissenschaftlichen Untersuchungen zur Gesundheitsversorgung ist vielfach auf die Bedeutung einer unterhalb der gesetzlichen Normierung angesiedelten intermediären Steuerungsebene hingewiesen worden (Döhler / Manow 1995). Im deutschen Sprachraum ist diese „Mesoebene“ (Braun et al. 2010: 41) schon länger Gegenstand der verwaltungswissenschaftlichen Implementationsforschung (Grunow 2006). Sie ist fester Bestandteil der mehrdimensionalen Infrastruktur des deutschen Wohlfahrtsstaats (Bode 2013) und gewährleistet dessen operationale Governance (Hill / Hupe 2009). Obwohl die Akteure dieser Mesoebene gegenüber ihren Bezugsgruppen (z.B. Berufstätigen oder Managern) eine von „der Praxis“ abgehobene repräsentative Sprecherfunktion einnehmen, bündeln sie maßgebliche normative und interes-

11 Diese Entwicklungen haben sicherlich auch mit Veränderungen in der Aufgabenumwelt (erhöhter Patientendurchlauf, kürzere Liegezeiten, mehr Aufwand pro Behandlungsfall) sowie mit veränderten Leistungserwartungen zu tun (Vogd 2011). Indizien für Letzteres sind die Durchsetzung des „Informed-Consent"-Prinzips oder die Entwicklung des Arzthaftungsrechts.

12 Pioniere solcher Reorganisationsstrategien waren die zuletzt deutlich expandierenden Privatkliniken (Heubel et al. 2010). 
senbezogene Orientierungen (Bode 1998). Die Akteursgruppen finden hier also einen wichtigen Ort für die Verarbeitung des Strukturwandels - und zwar auf der Grundlage verdichteter, auch Praxiserfahrungen entnommener, Deutungen, die in ein spezifisches „Steuerungsdenken“ übersetzt werden. Gewiss sind ihre Äußerungen immer auch von instrumenteller Rationalität geleitet - hinter einer politischen Strategie steht aber stets eine kognitiv ,sedimentierte“ Weltsicht auf das, was ein Ordnungsprinzip ist und sein soll.

Geht es also um Wettbewerb im Krankenhauswesen, versprechen Deutungsmusteranalysen auf dieser intermediären Ebene interessante Aufschlüsse über jenes Kräftefeld, in dem sich effektive Steuerungsprozesse bewegen. Nachfolgend rekapitulieren wir wettbewerbsbezogene Deutungsmuster bei fünf Akteursgruppen mit je spezifischen Rollen, nämlich: Zahler, Planer, Betreiber, ärztliche und nicht-ärztliche Berufsgruppen. ${ }^{13}$ Wir stellen zunächst die methodische Vorgehensweise vor, präsentieren dann die Argumentationsfiguren und präparieren aus diesen - auch vergleichend - die jeweils auf das Wettbewerbsprinzip bezogenen Elemente kognitiver Praxis heraus.

\section{Methode}

Die folgenden Befunde basieren v.a. auf Interviews mit VerbandsreferentInnen, bei denen ein Leitfaden eingesetzt wurde, der sowohl narrative Passagen generierte als auch Experteninformationen erhob (Scheibelhofer 2008; Liebold / Trinczek 2009). Kerngegenstand der Analyse sind 15 Gespräche, die mitgeschnitten, transkribiert und inhaltsanalytisch ausgewertet wurden. Kontrolliert wurde die Kohärenz der Interviewaussagen einerseits durch deren Abgleich mit Beschreibungen von Ko-Akteuren, andererseits durch die Sichtung von Schriftmaterial mit offiziellen Äußerungen aus den Akteursgruppen (Fachpresse, Internetportale, Verbandszeitschriften). ${ }^{14}$

Zur Gruppe der untersuchten Kollektivakteure gehörten: wesentliche administrative Instanzen (Krankenkassen und Länder), für den Patientenkontakt relevante Professionsgruppen (Ärzte und Versorgungspersonal) und die Betreiber (verschiedene Trägertypen sowie der zentrale Trägerverbund). Gefragt wurde nach dem Verständnis des real existierenden Wettbewerbs, ferner den von den Akteuren identifizierten Wettbewerbsproblemen sowie ihren $\mathrm{Be}$ wertungen im Hinblick auf die Regulierung des Wettbewerbs. Die Auswertung des Materials konzentrierte sich auf robuste Deutungsmuster, die inhaltsanalytisch qua Paraphrasierung und Verdichtung herausgearbeitet wurden. Auf diese Weise gelangen wir zur Komposition von Deutungsmusterkomplexen und zur Bestimmung von Zonen kognitiver Ambivalenz in der kognitiven Praxis der Akteure bzw. im gesamten Feld.

Wir präsentieren nachfolgend das Material, verdichtet in Gestalt synthetischer Argumentationsfiguren: Aussagen der Befragten einer Gruppe wurden als geschlossener Diskurs simuliert. Die Figuren, die nachfolgend für die verschiedenen Akteure rekapituliert werden, basieren dabei auf Interviewäußerungen und weiteren öffentlichen Stellungnahmen. Das Risiko von Fehldeutungen bestimmter Einzelaussagen wurde durch die Fokussierung der Auswertung auf große ,gemeinsame Nenner“ minimiert (wesentliche Diskursvariationen dokumentieren

13 Somit umfasst die Untersuchung wesentliche Infrastrukturebenen im Gesundheitswesen (Bode 2013). Politische Akteure strictu sensu (also partei- oder lagerpolitisch gefärbte Grundsatzdebatten) ziehen wir vor die Klammer, weil wir sie eher als Impulsgeber begreifen. Zwischen den Gruppen gibt es Überlappungen: Die Deutsche Krankenhausgesellschaft oder Ärztekammern agieren partiell auch als Vertreter von Berufsgruppen, sind in ihrer regulativen Rolle allerdings stark administrativ-körperschaftlichen Funktionen verpflichtet. Verbände von Leitungskräften repräsentieren einerseits eine Berufsgruppe (Manager), andererseits aber v.a. die Träger.

14 Im Rahmen des oben genannten Forschungsprojekts arbeiten wir mit einer Dokumentendatenbank, die den Zeitraum von 2009 bis heute abdeckt. 
nachfolgend die Fußnoten). Das Syntheseverfahren wurde auch deshalb gewählt, weil es eine Anonymisierung der Gesprächspartner ermöglicht. Verbandsnamen werden also nicht benannt; dadurch entstehende Unschärfen bei der Verortung der Akteure müssen in Kauf genommen werden. Das Sample beschreibt die Vielfalt der möglichen Deutungen sicherlich nicht erschöpfend. Auch kann im Rahmen dieses Beitrags keine streng hermeneutische Interpretation von Diskurspassagen angestrengt werden, für die man sehr viel mehr ins Detail einzelner Akteurskontexte gehen müsste. Wir nutzen Zitate eher zur Plausibilisierung unserer Inhaltsanalyse. Diese Analyse reicht in jedem Fall aus, um für die Vielfalt des bei sektorrelevanten Akteuren entwickelten „Wettbewerbsdenkens“ zu sensibilisieren und Implikationen zu diskutieren.

\section{Fünf Argumentationsfiguren}

\section{(I) Krankenkassen als Zahler}

Die Argumentationsfigur, die Vertreter der gesetzlichen Krankenversicherung entwickeln, erscheint relativ homogen: Überall gilt Wettbewerb als sinnvoll und alternativlos, um eine qualitativ bessere und zugleich wirtschaftlichere Versorgung zu gewährleisten - v.a. dann, wenn „mehr Vertragsfreiheiten“ geschaffen werden:

„,Wir brauchen mehr Wettbewerb - auch auf der Seite der Leistungserbringer. Und wir brauchen mehr Vertragsfreiheiten, um mit fortschrittlichen Ärzten und Kliniken eine gute und wirtschaftliche Versorgung für die Versicherten in der GKV zu schaffen."

„,Wettbewerb um medizinisch hochwertige Versorgungskonzepte verlangt freie Vertragspartnerwahl und echte Gestaltungsspielräume. "15

Allerdings erkennen die Kassen im gegenwärtigen Krankenhauswesen kaum Anbieterkonkurrenz; das DRG-System wird nicht (direkt) mit Wettbewerb assoziiert. Letzterer sei indes sinnvoll, denn er erhöhe bei den Leistungsanbietern die „Arbeitsaktivität, weil es sozusagen zu einer Überlebensfrage wird, inwieweit man Versicherte [...] attrahieren kann“. Ferner müsse man ,unterscheiden zwischen der Wirkung [des Wettbewerbs] für die Versicherten und die Wirkung für die dort Beschäftigten“. Für die sei es „nicht besonders erhebend, am Band zu stehen, aber es ist gut für die Gesundheit der Versicherten“.

Bestehende (Selektiv-)Verträge zur integrierten Versorgung deuteten - so ein Verbandsvertreter - auf eine „misslungene Deregulierung“ hin, weil sie zwar zu zusätzlichen Kontrakten geführt hätten, aber keine zählbaren Effekte aufwiesen.

Viele Äußerungen der Kassen zum Thema Wettbewerb beziehen sich auf das, was sie prospektiv für angezeigt halten. So werden auf Anbieterwettbewerb basierende Steuerungen als Alternative zum System der Landeskrankenhausplanung in Stellung gebracht:

„Da passiert ja nichts. Da gibt es ja keine empirische Untersuchung, da gibt es keine Qualitätssicherung, da gibt es keine Mindeststeuerung, da gibt es gar nichts. Da gibt es eine Excel-Tabelle, wo der die Betten fortschreibt für das nächste Jahr. Das ist meiner Ansicht nach total archaisch."

Wichtig sei die Entwicklung eines „organisierten Wettbewerbs“ bzw. die Etablierung eines Marktes mit obligatorischen Standards und Qualitätsrichtlinien, denn „nur mit bestimmten

15 Beide Zitate sind offizielle Positionierungen von Krankenkassenverbänden. Die Äußerungen beziehen sich auf das Gesundheitswesen als Ganzes. Wenn es um die stationäre Versorgung geht, zeigen sich gewisse Nuancen. 
Regeln führt der Wettbewerb auch zum Wohle der Versicherten“. ${ }^{16}$ Das Konzept unterscheidet dabei zwischen einer weiterhin kollektiv zu regulierenden Notfallversorgung und dem Bereich selektiver Leistungen (z.B. Knieoperationen), ,die man durchaus dem Wettbewerb überantworten kann" - gemeint sind Selektivverträge, durch die Krankenkassen Leistungspakete bei Kliniken einkaufen.

Qualität müsse dabei „transparent sein, gnadenlos [...]“. Die heutigen Qualitätsberichte der Krankenhäuser seien ein erster Schritt in diese Richtung; allerdings bestehe erheblicher Optimierungsbedarf:

„,Mehr Wissen um die Qualität der Leistung sorgt für mehr Wettbewerb und mehr Wirtschaftlichkeit. Das führt zu einer Win-Win-Situation aller Beteiligten. Und nur so können wir der Über- und Fehlversorgung Herr werden. [...] Wir müssen alles daran setzen, Leistungen auf dem Gesundheitsmarkt transparent werden zu lassen. [...] Vergleichen wir noch einmal mit dem Immobilienmarkt: Wir können uns ,Pfusch am Bau' nicht leisten. "17

Als zentrale Herausforderung begreift man das Monitoring von Versorgungsqualität in Kombination mit Preiswettbewerb. Hier möchte man selbst Regie führen, befindet sich aber offenbar noch auf der Suche nach der richtigen Methode:

„,Wir werden wahrscheinlich, wenn man Wettbewerb einführt, die Qualität irgendwie kollektiv messen müssen. [...] Damit wir beim Rabattwettbewerb nicht nur die billigen Preise haben, sondern damit auch die Qualität besser wird. Das kann man organisieren. [...] Und insofern würden wir uns mit als Organisator eines solchen Wettbewerbs sehen."

„Im bisherigen System [...] spielt die eigentliche Qualität als solche überhaupt keine Rolle.

[...] Wir sind jetzt eher in der Situation, dass wir versuchen, [...] ein Messinstrumentarium aufzubauen."

In gewisser Hinsicht vertraut man auch auf die Selbstheilungskräfte des Marktes. Versicherte werden als informierte Konsumenten begriffen, die - ebenfalls miteinander konkurrierenden - Krankenkassen als ihre Agenten. Die Kassen sind „Auswahlprofis“ und „unterstützen“ die Patienten bei der Auswahl von Versorgungseinrichtungen nach Maßgabe von Qualitätsbewertungen. Versagten die Auswahlprofis, sei Abwanderung die Folge: „Wenn die Kasse einen Fehler dabei macht - zum Beispiel dann irgendwie immer das [betont] Todeskrankenhaus [betont] wählt oder das verschimmelte Krankenhaus - dann wird sie natürlich am Markt sanktioniert."

Gleichzeitig geht es um aktive Patientensteuerung. Man wisse ja nicht, ob die Versicherten medizinisch qualitätsbewusst seien oder „ob nicht andere Punkte im Vordergrund stehen“. Das „subjektive Empfinden“ der Kassenklientel ist im Hinblick auf „Kostensteuerung nicht prioritär: „Wenn das Zimmer nicht schön ist, und ich sag das der Klinik, dann wollen die mehr Geld für Renovierungen. Da kann man nur sagen: Gehen Sie zukünftig in die und die Kliniken.“

Insgesamt begreifen die Kassen Wettbewerb als zentrales Momentum zur Reorganisation der Krankenhausversorgung, wobei Qualitätsrisiken als beherrschbar gelten. Er wird in Stellung gebracht gegen sämtliche Ko-Akteure des Gesundheitswesens mit dem Ziel, die Kassen

16 Im Kassenwesen zeigen sich bestimmte Nuancen im Hinblick auf das gewünschte Verhältnis von Kollektiv- und Selektivverträgen. Während die Konzepte des GKV-Spitzenverbandes und des AOKBundesverbandes der hier dargestellten Position entsprechen (Leber 2004), versteht man beim VDEK Selektivverträge eher als ,,sinnvolle Ergänzung zum Kollektivvertrag“ (Ballast 2011).

17 Das Zitat entstammt einer Rede des Vorsitzenden der Innungs-Krankenkasse auf der „Plattform Gesundheit" des IKK e.V. im Oktober 2011. 
zum „Gestalter“ und Agenten „zum Wohle der Versicherten“ zu machen. Inwieweit die sehr plakativen Forderungen letztlich eher strategischer Natur sind, muss hier offen bleiben. ${ }^{18}$

\section{(II) Länder als Planer}

Fundamental für die Argumentationsfigur, die wir für die Gruppe der Landesakteure rekonstruieren, erscheinen die Sicherstellung der „Grund- und Notfallversorgung“ sowie eine dahingehende Planungskompetenz. Wettbewerb wird allgemein gedeutet als „Politik der Häuser“, die sich ,zu echten Wirtschaftsbetrieben entwickeln“ müssen. Was die Folgen anbelangt, so gebe es „Patientenwanderungen zwischen den einzelnen Regionen“, auch im Zusammenhang mit Qualitätsaspekten. Die Frage sei dabei: „Wer verkauft sich besser, wer investiert mehr in seine Qualität, wessen Ruf.“ Allerdings sei „die Transparenz des Ergebnisses herzustellen [...] ungeheuer schwierig im Krankenhausbereich.“

Qualitätswettbewerb gilt allgemein als sinnvoll, Verdrängungswettbewerb aber bereitet Schwierigkeiten; die „unselige[n] Zuweiserprämien“ sowie Kapazitätsausweiterungen zur Markterschließung sind problematische „Auswüchse“.

„Das ist manchmal der [...] etwas kurzsichtige Wettbewerb, Mann gegen Mann. Ich schaffe jetzt einen Schwerpunkt, den das Nachbarkrankenhaus schon hat, und versuche, denen die Fälle wegzunehmen. Und wenn der Chefarzt dann wieder geht, dann falle ich wieder hinten runter. [...] Das kann letztlich keinen nachhaltigen Erfolg haben. "

Destruktiver Konkurrenz will man entgegenwirken: „Das Land achtet darauf, dass die bestehenden Standorte auch weiter ausgelastet sind, und wenn es was Neues gibt, das tatsächlich auch was Neues sein muss.“ Wichtig sei die Arbeit in „regionalen Netzwerken“, in denen man sich auf Versorgungsstrukturen einigt. Ex-Post-Kontrolle durch Marktregulierer (wie den Medizinischen Dienst der Krankenkassen) habe Grenzen und könne zu übermäßigen „Prüforgien“ führen.

Dort, wo Krankenkassen durch Selektivverträge mehr (Preis-)Konkurrenz entfachen wollen, erkennt man Tendenzen, die die Krankenhauslandschaft und die dort bestehenden „Aushandlungswege“ unübersichtlicher machen. Man beobachte, dass dies „nicht wirklich zur Kostensenkung auf Dauer beitragen würde“. Als Anschauungsbeispiel gelten nicht-stationäre Anbieter einbeziehende Integrierte Versorgungsverträge, die Krankenkassen schon jetzt marktstrategisch nutzten:

„,Das führt letztlich zu [...] schwarzen Krankenhäusern [...] [und] Rosinenpickerei. Das treiben einige Kassen noch bunter als andere. Weil sie natürlich damit auch wahrscheinlich ein Preisdumping verbinden. Ob das qualitativ so gut ist, weiß ich nicht. " 19

Wettbewerb könne „,in Zukunft“ aber als Modellkonkurrenz funktionieren: „Eine Region versucht besser zu sein als eine andere, [eher] als ein Krankenhaus in einer Region gegen ein

18 Für Paquet (2011) schrecken die Kassen vor einer vollständigen Ersetzung des Kollektivvertrags durch Selektivverträge zurück. Vielfach gehe es eher um „Schaufensterwettbewerb“ (Paquet 2011: 41).

19 Man bezieht sich hier auf Integrierte Versorgungsverträge, durch die Arztpraxen stationäre Aufgaben übernehmen. Die Bewertung variiert im Akteursfeld ein wenig: So kann sich ein Befragter eine vertragspolitische „Öffnung auf Augenhöhe“ unter Berücksichtigung von „Entfernungsqualitäts- und Erreichbarkeitsparametern“ durchaus vorstellen. 
anderes.“ Akzeptabel sei Wettbewerb als Instrument zur Verdrängung überzähliger Krankenhäuser, aber nur für Bereiche abseits der Grundversorgung und ohne deren Gefährdung. ${ }^{20}$

Die oben genannte leistungsorientierte Investitionsförderung (die die Krankenhausförderung an deren Wettbewerbserfolge koppelt) will man durch ein „Dialog-gestähltes Indikatorenset“ und unter Berücksichtigung von Grundbedarfen kontrollieren, „um auch Häuser mit in der Förderung zu haben, [...] bei denen das Wasser in die Notaufnahme läuft, also wo es noch tatsächlich Investitionsbedarf gibt." Eine gemischte Investitionsförderung (teils nach Maßgabe von „Case-Mix“-Größen, teils auf Einzelantrag) gilt als solide Lösung.

In der Auseinandersetzung um zukünftige Grenzen des Wettbewerbs spielen Begriffe wie wohnortnah und Bedarf eine zentrale Rolle - wobei der entsprechende Diskurs vage bleibt. Die Devise laute:

„, Notfallversorgung sichern, dazu auch eine gewisse Grundversorgung, und diese Standorte möglichst auch dauerhaft erhalten. Und über den ganzen Rest im Krankenhausbereich, da darf es durchaus auch einen Wettbewerb geben, [um] [...] die Versteinerung in der Landschaft insgesamt aufzuheben. [...] Das ist letztlich die Aufgabe, die wir haben im Rahmen der Krankenhausplanung, und insofern haben wir auch diese - für mich immer hohlenBegriffe, bedarfsgerechte Versorgung, ortsnahe Versorgung, konkretisiert. [...] Wir versuchen planerisch darauf hinzuwirken, dass wir gucken, wo sind denn die Bedarfe, die tatsächlich in einer Grundversorgung in guter Qualität vorhanden sein müssen. Und da kommen wir zu dem Punkt, dass die Geburtshilfe möglicherweise nicht mehr dazu gehört. "

Kurzum: Es gilt das Motto „Wettbewerb ja, aber...“ In ihrem Selbstverständnis als „Planer“ suchen Bundesländer gleichsam tastend Strukturen, in denen die Grundbedarfsorientierung des Krankenhauswesens gewahrt und Wettbewerb eher arrondierend wirkt. Die Rückzugsposition ist dabei die Sicherstellung von wettbewerbsfreier (ggf. sektorübergreifender) „Grundversorgung".

\section{(III) Träger als Operateure}

In der Diskursfigur, die sich für die Gruppe der Krankenhausträger rekonstruieren lässt und sowohl Träger- als auch Managerverbände repräsentiert, ist Wettbewerb als allgemeine Steuerungsidee ,durchaus nachvollziehbar“: ${ }^{21}$

„In ein Krankenhaus, in dem ich gut versorgt werde, da gehen die Leute auch gerne hin, da kommen dann auch genügend Patienten, die können dann eben auch ihre Kapazitäten auslasten und können dann auch wirtschaftlich arbeiten, und Häuser, die an dieser Stelle schlechte Qualität liefern, da stimmen die Patienten dann auch mit Füßen ab. “

Für aussichtsreich befunden wird dabei insbesondere die Ausweitung eines intersektoralen Wettbewerbs (mit den ambulanten Fachärzten), da es „für das Gesundheitswesen einfach auch effizient wäre, wenn hier eine Öffnung stattfände“.

Für die Träger scheint es ausgemacht, dass Krankenhäuser heute „,strategisch denken müssen, auch überlegen müssen, wie in der Industrie, welches Portfolio biete ich an“. Als Ziel-

20 Einige Unterschiede in den Einstellungen der Länder zu Wettbewerb und Selektivverträgen lassen sich mit bundeslandspezifischen Besonderheiten (z.B. der Bevölkerungsdichte) erklären.

21 Die hier präsentierte Diskursfigur blendet Stellungnahmen privat-gewerblicher Träger aus - allerdings wurden Verbände befragt, die auch Akteure aus diesem Lager vertreten. Laut Paquet (2011: 41) votierten diese Träger strategisch „fast alle für die Beibehaltung des Status quo“. 
gruppe gelten weniger die Patienten als die einweisenden Ärzte. ${ }^{22}$ Wettbewerb um „Mengen“ (von Patienten) sei gängige Praxis, die Häuser selbst widmeten sich vor allem einer Frage: „Wie kriege ich [...] die Patienten in mein Haus, anstatt dass sie zum Nachbarn gehen.“ Im bestehenden Wettbewerb müsse aktiv gehandelt werden; entsprechend würden Qualitätsausweise wie Zertifizierungen ,sehr stark genutzt, um sich da einen gewissen Wettbewerbsvorteil erstmal zu schaffen“.

Die Realität des DRG-Systems wird häufig auf der Folie von Erfahrungen mit einer direkteren „Staatsplanung“ (z.B. über Budgetdeckelungen) interpretiert. So sei z.B. die (dem DRG Modell nachempfundene) „leistungsorientierte Investitionsfinanzierung“ „fairer“ als die „Gutsherrenmentalität“ der Länder.

Die real existierende Trägerkonkurrenz gilt indes durchaus als problembehaftet. Denn beim „Wettbewerb ist man eigentlich vor allem auch so den eigenen Interessen verantwortlich und nicht so sehr der Gesellschaft" - in den Kliniken sei das heute spürbar:

„Jeder, der einen höheren Aufwand verursacht, das macht Ihnen keine Freude. [...] Also da kommt ein Geist dann ins Krankenhaus rein, der da nicht gut ist, der [...] dieser unbedingten Verpflichtung dem Patienten gegenüber [...] abträglich ist. Also es, da ist so was Dehumanisierendes drin. "

Man sieht zumindest Tendenzen hin zu einem „Verdrängungswettbewerb [...,] der auf die Substanz geht“ und - im Kontext von Überkapazitäten und Fehlinvestitionen - „, mit Sicherheit schon eine Gefahr“ darstelle. Diagnostiziert wird, dass gelegentlich „Aussieben, Pickerei betrieben wird“", weil eine bestimmte Leistung nicht wirtschaftlich erbracht werden könne.

Als bedenklich gelten insbesondere ,wettbewerbsverzerrende“"Versuche von Kliniken, Patienten durch Leistungen ,im Servicebereich“ anzuwerben und gleichzeitig schlechtere fachliche Qualitäten zu bieten. Die dabei genutzten Informationsinstrumente hätten begrenzte Aussagekraft; für den Patienten sei die „eigene Erfahrung oder die Erfahrung des Nachbarn wichtiger" als das Informationsangebot offizieller Qualitätsberichte. Letztere seien oft eine unzureichende Informationsgrundlage - und „Show-Effekte“ die Folge,

„, weil man da ja auch unheimlich manipulieren kann. Jetzt, wenn sie eine schöne große Palliativeinheit haben und wissen jetzt in der Inneren, der wird jetzt morgen sowieso sterben - also klingt jetzt brutal: die Wahrscheinlichkeit, den zu retten ist gering - dann wird da halt noch einen Tag vorher verlegt. "

Insgesamt wird für einen öffentlich regulierten Qualitätswettbewerb plädiert:

„, im Sinne der Daseinsvorsorge und demokratisch legitimiert sollten [...] entsprechende Gremien beschließen: Wir wollen Versorgung für jedermann auf diese Art und Weise [...] gestalten. Und in, wenn man dann so eine [...] Infrastruktur hat, [...] dann ist es durchaus okay, wenn man gerade über den Qualitätswettbewerb das sich weiter entfalten lässt. "

Die zentrale Herausforderung bestünde dabei darin, Wettbewerb hier „so zu gestalten, dass auch Bewertbarkeiten entstehen“ und dass „eben nicht nur tot oder lebendig berücksichtigt wird, sondern [...] Qualität eben auch noch aus anderen, weicheren Faktoren besteht“". Gleichzeitig sieht man praktische Hindernisse, jedenfalls müsse ,wegen des Aufwands“ Komplexität „radikal reduziert“" werden. Bestehende Richtlinien zur Qualitätssicherung („Mindestmengen, Strukturqualitäten") werden kritisiert, da es für die damit verbundenen Anstrengungen (z.B. Hygienefachkräfte oder Fachärzte für Neurologie) „keine Refinanzierung“ gebe. Auch werde

22 Es findet sich teilweise aber auch die Einschätzung, ,dass der Qualitätswettbewerb das ist, [...] wonach die Patienten in Zukunft auch aussuchen werden“. 
der Pflegeaufwand in den DRGs nicht hinlänglich abgebildet. Die vorherrschenden Qualitätsprüfungen seien oft überbürokratisch.

Verbreitet zeigt sich Skepsis im Hinblick auf die Messbarkeit von Qualität sowie der Kontrolle von Umgehungsstrategien, zumal im Hinblick auf Konzepte, die die Trägerfinanzierung an Qualitätsergebnissen ausrichten wollen:

„,Wenn Sie mir sagen können, was Performance ist, und dass es auch tatsächlich dann Performance ist, dann kann man auch über differenzierte Vergütung nachdenken. [...] Ja, denn das ist doch das Problem, dass man das medizinisch kaum greifen kann. [...] Die Kassen wollen einfach nur das Einkaufsmodell durchsetzen. "

Preiswettbewerb findet wenig Befürworter. Ein hoher „Vertragsverwaltungsaufwand“, eine Zunahme von Qualitätsprüfungen, eine „Ungleichbehandlung“ aufgrund der Abhängigkeit wirtschaftlicher Verhältnisse vom Verhandlungsgeschick Einzelner sowie die Einschränkung der „freien Krankenhauswahl“ gelten als potenzielle Auslöser „größerer Verwerfungen“. Das Ergebnis seien „Dumpingpreise [...] und das kann [...] dann auch wirklich gefährlich werden, wenn man das in die Medizin hineinbekommt" ${ }^{23}$

Kurzum: Qualitätswettbewerb als probates Distinktionsfeld für die einzelnen Krankenhäuser, aber für das Gesamtsystem schwer operationalisierbar oder gar gefährlich, wenn er mit dem Finanzierungssystem (zu) kurz geschlossen wird - so könnte man die Perspektive der Klinikbetreiber zusammenfassen.

\section{(IV) Ärzte als beruflich Involvierte}

Die für die organisierte Ärzteschaft rekapitulierte Argumentationsfigur basiert auf einer Schnittmenge von Äußerungen aus Berufsverbänden bzw. Kammern, die sich auf den Krankenhaussektor beziehen. ${ }^{24}$ Grundlegend ist hier eine relativ große Skepsis gegenüber Wettbewerb. Diese beruht darauf, dass man es - ,anders als im klassischen marktwirtschaftlichen Wirtschaftssystem" - mit einem Bereich zu tun habe,

„,der sich einem Wettbewerbsprinzip als Ordnungs- oder Steuerungsprinzip [...] aus den verschiedensten Gründen verschließt. [...] Da kommt für mich eigentlich das Credo, dass es im Krankenhausbereich [...] keinen Preiswettbewerb geben darf, sondern, wenn überhaupt, über Elemente wie die Qualitätssicherung einen Leistungswettbewerb. “

Problematische Folgen seien schlechte Arbeitsbedingungen, eine schädliche Konkurrenz um Patienten und falsche strategische Weichenstellungen der Kliniken. Letzteres bezieht sich auf die Ausdünnung des Leistungsspektrums bzw. die Angebotsspezialisierung, was im Übrigen auch die (Facharzt-)Weiterbildung erschwere,

„, weil die Ärzte, die die jungen Leute weiterbilden sollen, also sprich die Oberärzte und die Chefärzte, natürlich in genau demselben Problem mit im Hamsterrad stecken wie alle an-

23 Einige Akteure, insbesondere die privat-gewerblichen Träger, zeigen sich hier offener - jedoch nur bei fortbestehender Sicherstellungsverpflichtung und ohne „freie“ Preisverhandlungen. Insgesamt herrscht aber die Überzeugung vor, dass , der Preiswettbewerb letztendlich zu Lasten der flächendeckenden Versorgung gehen würde, dass die Krankenhausplanung ausgehebelt wird, dass die Krankenhäuser gegenseitig ausgehebelt werden - weil die Marktmacht dann bei den Krankenkassen liegt“".

24 Die Ärzteverbände fordern allgemein weniger staatliche Regulierung und mehr Wettbewerb bzw. größere Autonomie für die Selbstverwaltung - zumeist hinsichtlich der ambulanten Versorgung (so in der „Allianz deutscher Ärzte“ als Zusammenschluss verschiedener Verbände wie Hartmannbund, NAV-Virchow Bund u.a.). 
deren, so dass also vielfach keine Zeit dafür bleibt, wirklich Dinge zu vermitteln, wie man das normalerweise machen müsste".

Selektive Angebotsplanung trete an die Stelle der traditionellen Orientierung an umfassender Patientenversorgung, man müsse jetzt „,öllig andere Gesichtspunkte“ in die medizinische Praxis einbringen. Folglich würden die Ärzte in ihren Entscheidungen und Handlungen irritiert:
„Heute ist es so, sie müssen eben als Krankenhaus irgendwelche Leistungen planen, sie müssen sich strategisch aufstellen, sagen, das will ich in Zukunft gar nicht mehr machen, und wenn der Patient dann kommt, dann schicke ich ihn woanders hin, oder ich biete ir- gendwelche Dinge erst gar nicht mehr wieder an und mache auf diese Weise, also selektiere ich praktisch mein Leistungsspektrum. Und das ist an sich eine Denkart, die Ärzten so per se fremd ist. "

Pläne, das Krankenhauswesen mit Selektivverträgen zu steuern, führten zu einer Zuspitzung der allgemeinen Tendenz. Die Versorgung dürfe „nicht mit Prinzipien“ einer „kommerzielle[n], industrialisierte[n] Leistungserbringung“" gesteuert werden, denn die damit verbundene Unterbietungslogik bzw. das „Preisdumping“ laufe darauf hinaus, ,,dass Leistungen gemindert werden“. Den Chefärzten, denen man ein Budget gebe, bedeute man: „Du musst jetzt gucken, wie du damit klar kommst. Aber [...] er muss es ja auch irgendwo weitergeben, also [...] letzten Endes treffen wir natürlich auch die Patienten damit."

Man müsse sich dessen ungeachtet als Profession auf die neuen Rahmenbedingungen einstellen. ${ }^{25}$ Qualitätswettbewerb wird akzeptiert, aber nicht offensiv als Ansatz zur Marktregulierung vertreten. Qualitätssicherung gilt als Ärztesache und nicht als ein Mechanismus, den medizinexterne Akteure (allen voran die Krankenkassen) mitgestalten sollten. Angemerkt wird, dass ,natürlich die Ärzte grundsätzlich das schon auch als ärztliche Aufgabe ansehen, dass Qualitätssicherung gemacht wird“. Man äußert allerdings Zweifel bezüglich der Vorstellung, dass sich die bestehenden Konkurrenzverhältnisse in Richtung Qualitätswettbewerb bewegen:

„Das ist immer so die hehre Vorstellung, dass Wettbewerb sich immer auf der Ebene des Qualitätswettbewerbs sich abspielt; aber man muss natürlich ganz klar [...] sehen, dass es sehr häufig eben doch um einen, [...] nicht immer einen Preiswettbewerb, aber [...] schon um einen existentiellen Wettbewerb [geht]. "

Kurzum: Die organisierte Ärzteschaft kann sich mit Wettbewerb als Steuerungsprinzip kaum anfreunden und assoziiert mit ihm letztlich eine Ökonomisierung medizinischer Praxis. Man wendet sich nicht prinzipiell gegen einen Wettbewerb um Qualität - aber dieser gilt als technisch delikat und gehört keinesfalls in fremde Hände.

\section{(V) Versorgungskräfte als beruflich Involvierte (Pflege und Sozialarbeit)}

Die Argumentationsfigur, die die Perspektiven von Repräsentanten der Versorgungskräfte zusammenfasst, stellt eine Synthese gewerkschaftlicher und berufsverbandlicher Haltungen dar. Allgemein zeigt sich hier eine vorsichtige Offenheit gegenüber wettbewerblicher Steuerung

25 Man will sich die im neuen (Wettbewerbs-)Umfeld relevanten Wissensbestände aneignen, um mitreden zu können (dies legt auch das in dieser Hinsicht ambitionierte „Curriculum ärztliche Führung“ der Bundesärztekammer nahe). 
im Krankenhauswesen. ${ }^{26}$ Festgemacht an der Einführung der DRG-Finanzierung, habe diese eine „Straffung der Prozesse ausgelöst“ sowie die „Änderung von Organisations- und Ablaufstrukturen“, auch das Aufbrechen bestehender Machtverhältnisse, hier v.a. der „Dominanz der Medizin“. Auch eine stärkere Konzentration auf das Wohlergehen der Patienten wird mit dem bestehenden Wettbewerbssystem assoziiert: Letzteres zwänge „Krankenhäuser, das wirklich ernst zu nehmen, weil sie sich nur so im Wettbewerb behaupten können.“ Das Qualitätsmanagement der Kliniken sei ebenfalls optimiert worden, denn dies sei „die einzigste Chance, um im Wettbewerb bestehen zu können". 27

Wettbewerb wird nicht zuletzt mit einer möglichen Neuordnung der krankenhausinternen Arbeitsteilung in Verbindung gebracht.

„Wenn sich schon alle Krankenhäuser im Wettbewerb, im harten Verdrängungswettbewerb
untereinander befinden, [dann] sind die Geschäftsführer natürlich gezwungen, ganz genau
zu überprüfen: Was brauche ich zwingend, um die Behandlung des Patienten sicherzustel-
len [...]. Das heißt, in den Krankenhäusern geht auch die Debatte darum, welche Berufs-
gruppe macht eigentlich was?"

Hier zeigen sich Hoffnungen auf Machtverschiebungen innerhalb der Krankenhäuser. Die Versorgungskräfte sehen sich als Promotoren des Wandels, als Träger von Innovationen, die Prozesse „schlanker und schneller“ und „kostengünstiger“" machen.

„, [Man] kann dann noch mal vor dem Hintergrund der Effizienz und auch der größten Kompetenz oder auch der größten Patientennähe, oder Nähe am Betroffenen, entscheiden, wer sollte das dann sinnigerweise tun? "

„,Wir sehen die Unternehmersicht, [...] indem wir die Prozesse neu gestaltet haben, indem wir gesagt haben, wir übernehmen ärztliche Aufgaben. "

Allerdings werden Risiken einer neuen Arbeitsteilung thematisiert, v.a. der Verlust an Ganzheitlichkeit und neue Schnittstellenproblematiken. "28

Gleichzeitig provoziert die Trägerkonkurrenz, so eine typische Diagnose aus dem Kreis der fraglichen Akteursgruppe, ${ }^{29}$ in den Kliniken das Aufkommen einer bestimmten Geschäftslogik: „Was kann ich unter wirtschaftlichen Aspekten am Besten, wo bin ich der Player?“ Man konzentriere sich auf „,das Feld der Privatpatienten, [...] weil die natürlich Geld bringen“, suche nach ,attraktiven Eingriffen, [...] die man in das eigene Haus binden will“ - , und andere, die vernachlässigt man dann einfach“. Besonders Preiskonkurrenz setze eine problematische Dynamik in Gang; sie provoziere „Dumpingwettbewerb“ sowie eine Erwartung: ,gleiche Leistung für $[\ldots]$ weniger Geld“. Der ,positive Effekt" von Wettbewerb werde dann „,natürlich konterkariert". Allgemein ließen sich viele Krankenhäuser zunehmend vom Markt treiben: „Wir beobachten, dass Strategien der Kliniken oder der Klinikführungen inzwischen immer kurzfristiger werden; also mittel- bis langfristig an Planung sieht man eigentlich wirklich nachhaltig kaum noch.“

Bei der Reflektion von Folgen der Anbieterkonkurrenz wird überdies über Stellenabbau und Arbeitsverdichtung geklagt. Der heutige Wettbewerb im Krankenhauswesen drehe sich, so der

26 Beim gewerkschaftlichen Vertreter ist die Offenheit eher hypothetischer Natur. Er sei „grundsätzlich kein großer Freund von Wettbewerb“, kann sich ihn aber ,,vorstellen, wenn er vernünftig organisiert wird“ - denkbar seien mehr Kostentransparenz und „möglicherweise“ eine „effektivere Organisation".

27 Es gibt zudem eine gewisse Offenheit gegenüber Selektivverträgen bei der sogenannten Integrierten Versorgung (die ja im Rahmen eines Preiswettbewerbs abgeschlossen werden).

28 Manche Stimmen plädieren (mit Blick auf die Pflege) dafür, ,dass wir vom ganzheitlichen Ansatz runter gehen müssen“ - also für mehr Arbeitsteilung innerhalb der fraglichen Berufsgruppe.

29 Die Diagnosen zum Ausmaß von Konkurrenz variieren allerdings zwischen den Akteuren. 
Tenor, vielfach nicht um Qualität: „Er geht um Lohndumping, [...] um schlechtere Arbeitsbedingungen. Man sieht eine „Dominanz der Ökonomie“, durch die die Pflege „überhaupt keinen Spielraum mehr" besitze.

Qualitätssicherung wird zwar als wichtiges Regulativ begriffen: „Ich glaube, dass die Häuser alle Bereiche einer qualitativen Evaluierung unterziehen sollten, weil ich glaube, das dient der Verbesserung [...] der Patientenversorgung." Vor einer Überschätzung dieses Regulativs wird allerdings gewarnt: „Ich glaube, viele Kunden orientieren sich nach wie, viele Patienten orientieren sich nach wie vor noch an Mund-zu-Mund-Propaganda."

Kurzum: Wettbewerb macht für diese Gruppe durchaus Sinn, wenn er sich für berufspolitische Zwecke nutzen lässt - er ruft aber Skepsis hervor, sobald davon abstrahiert wird.

\section{Zonen der Ambivalenz}

Wie gesehen, entwickeln die intermediären Akteure des Krankenhauswesens eine recht unterschiedliche kognitive Praxis im Hinblick auf das Wettbewerbsprinzip. Um einen oberflächlich unisono in Anschlag gebrachten Ordnungsbegriff herum bilden sich mehrere Zonen der Ambivalenz. Erstens wird die Deutung dessen, was Wettbewerb impliziert, mit je spezifischen Interesseninterpretationen verschränkt. So sehen die Versorgungskräfte Chancen, den entstehenden Konkurrenzdruck auf die Kliniken zur Verbesserung ihrer krankenhausinternen Machtposition zu nutzen, v.a. gegenüber der Ärzteschaft. Diese wiederum wehrt sich gegen eine wettbewerbsinduzierte „Außensteuerung“ durch Kostenträger, aber auch durch interne Kräfte, die den Druck Letzterer an sie weiterreichen (könnten). Divergenzen anderer Art gibt es zwischen Kostenträgern und Leistungserbringern. Die Krankenkassen wollen Käufermacht, die die Betreiber wiederum fürchten. Auch die Länder argumentieren im Lichte einer spezifischen Interesseninterpretation: Sie wollen Steuerungshoheit bewahren und nicht an die Krankenkassen abgeben, die sie vorwiegend als ,payer“ sehen.

Viele Akteure verknüpfen Wettbewerb gleichsam idiosynkratisch mit Nebenprojekten (Aufwertung von Berufsrollen, Funktionserweiterung vom ,payer zum player“, Aufbrechen von Sektorgrenzen, Vermeidung von Staatsplanung nach „Gutsherrenart“ etc.). Es geht dabei immer auch um Interpretationen der Interessen anderer, welche den eigenen Projekten im Wege stehen. Länder und Betreiber monieren die Tendenz von Kassen, unter dem Deckmäntelchen von Qualitätswettbewerb Einkaufspolitik zu betreiben. Letzteres sehen auch die Berufsgruppen. Der Fall des Versorgungspersonals zeigt, dass wegen der Nebenprojekte zuweilen ein großer Spagat vollzogen wird, in diesem Fall zwischen der - streckenweise fundamentalkritischen - Problematisierung eines Versorgungsziele gefährdenden Konkurrenzgebarens einerseits und den Chancen, Machtansprüchen der Ärzteprofession entgegenzutreten, andererseits. Ähnliches zeigt sich bei der affirmativen Bezugnahme der Betreiberverbände auf die Marktdistinktionsanstrengungen von Mitgliedsunternehmen; loyale Interessenvertretung ist hier das Nebenprojekt, welches sich schlecht mit aus der Vogelperspektive vorgetragener Sorge um die Folgen von Überbietungskonkurrenz verträgt. Schon in der Interessenperspektive also wird Wettbewerb auf der Ebene des (intermediären) Feldes sehr uneinheitlich gedeutet.

Zudem fällt auf, dass sämtliche Akteure je spezifische Rückzugspositionen einnehmen, wenn es darum geht zu bestimmen, wo und wie weit die „Freiheit“ des Wettbewerbs greift. Die Länder wollen Angebotsvernetzung und Wettbewerb gleichermaßen, Letzteren aber faktisch nur in der oben genannten Benchmarkfunktion. Für das Versorgungspersonal macht Konkurrenz zwischen Kliniken Sinn, aber nur dann, wenn sich der eigene Berufsstand dabei verbessern kann. Die Ärzte billigen (,wenn überhaupt“) Qualitätswettbewerb in Eigenregie, um ihre professionelle Autonomie (so gut wie möglich) zu wahren. ${ }^{30}$ Die Betreiber befürwor-

30 Wobei sie offenbar hoffen, dem Anbieterwettbewerb durch Qualitätssicherung in Eigenregie den Zahn ziehen zu können. 
ten Distinktionswettbewerb, verweisen aber - ähnlich wie Versorgungskräfte und Planer (Länder) - auf Probleme einer Steuerung, bei der die Erfüllung bestimmter (formaler) Qualitätsstandards durch Kostenträger finanziell ,belohnt“ wird; möglicherweise beruht ihre Skepsis auf Erfahrungen einer Basis, für die die Erzeugung von „Show-Effekten“ (z.B. bei der Bewerbung eigener Qualitäten) Tagesgeschäft ist. In ihrem Plädoyer für „gnadenlose“ Transparenz halten die Kassen - mit demonstrativem Steuerungsoptimismus - dagegen, obwohl durchscheint, dass praktikable Lösungen für ihr „Qualitätseinkaufsmodell“" noch nicht vorliegen. Die Kassen als ,Zahler“ wollen Wettbewerb und Organisation - dabei ist die Wahlfreiheit von Patienten nicht unbedingt prioritär bzw. nur dann, wenn ihnen das Wahlmenü durch die Kassen vorgegeben werden kann.

Schließlich existiert Uneindeutigkeit auch im Hinblick auf die offensichtliche „lingua franca“ des gegenwärtigen Krankenhauswesens, deren Kernbegriff der des Qualitätswettbewerbs ist. Nahezu sämtliche Akteure beziehen sich affirmativ auf diesen Terminus, meinen dabei aber meist jeweils etwas anderes. Die Kassen kaprizieren sich gleichsam furios auf Optionen, Qualitätswettbewerb als mit Geld hinterlegte Leistungskonkurrenz zu etablieren. Die Betreiber können der Vorstellung von Qualitätswettbewerb so lange folgen, wie sie über die oben genannten Distinktionsstrategien ihrer korporativen Mitglieder sprechen; Folgen auf der überbetrieblichen Ebene thematisieren sie kritisch als „Verdrängungswettbewerb“. Ärzte und Versorgungskräfte wollen sich dem Qualitätswettbewerb nicht verweigern, wenngleich diese Zustimmung eher nolens volens erteilt wird. Die Länder verstehen einen solchen Wettbewerb als „Benchmarkkonkurrenz“, z.B. zwischen Regionen, die die Substanz des Versorgungsgeschehens unberührt lässt.

Das allgemeine, aber diffuse Plädoyer für Qualitätswettbewerb spiegelt sicherlich die nach wie vor bestehende Geltung des Bedarfsprinzips wider. Das, was viele als „Verdrängungswettbewerb" bezeichnen, gilt - wenn es nicht (wie bei den Kassen) dethematisiert wird - als „Verzerrung“ oder „Auswuchs“. Die Anbieterkonkurrenz darf - so viel scheint Konsens - für niemanden die Versorgung gefährden. In der Sache bleibt damit jedoch ein bestimmtes Maß an Ambivalenz erhalten, denn der Wettlauf um gute Qualität impliziert, dass letztere hier mäßig und dort exzellent, also sektorübergreifend systematisch ungleich ist. Unter Ausblendung dieses Umstands können die Akteure indes dieser Interpretation von Wettbewerb folgen. Alles andere erzeugt bei ihnen zumindest Irritationen - zumal wenn sie, so wie die Bundesländer mit ihrem Sicherstellungsauftrag oder die Ärzte in ihrer Ausbildungsfunktion, Ergebnisverantwortung übernehmen (müssen).

\section{Schlussfolgerungen}

Wettbewerb im Krankenhauswesen gilt heute verbreitet als unumgänglich, wird aber bei für die operationale Governance des Krankenhaussektors maßgeblichen Akteuren mit verschiedenartigen Vorbehalten versehen. Spielräume, Kontroll(form)en, Grenzen, Gegenstände des Wettbewerbs - all dies wird ebenso vielstimmig wie spannungsgeladen reflektiert. Einerseits vermitteln idiosynkratische Interesseninterpretationen und die je spezifischen, untereinander weitgehend inkompatiblen Rückzugspositionen eher den Eindruck eines Burgfriedens denn eines allgemein geteilten Verständnisses von dem, was Wettbewerb im Krankenhauswesen ist und was er sein soll. Andererseits aber verschwimmen die kognitiv prozessierten, sich widerstreitenden institutionellen Logiken in der gemeinsamen (diffusen) „lingua franca“ des Qualitätswettbewerbs. Die kollektive Akzentuierung des Begriffs verweist zugleich auf die Persistenz der institutionellen Logik des Bedarfsprinzips als institutionelle Kernlogik eines wohlfahrtsstaatlich organisierten Gesundheitswesens, die von vielen (nicht allen) Akteuren gegenüber der institutionellen Logik der ,,business-like health care“ in Stellung gebracht wird. Selbst 
dann, wenn der Bezug auf das Bedarfsprinzip strategischer Natur sein sollte, sind die Geltung des Prinzips und insoweit auch dessen kognitive Internalisierung vorausgesetzt.

Zwar ist eine konsistente Vorstellung von dem, was der Markt im Krankenhauswesen leistet bzw. leisten kann, (noch) nicht zu erkennen. Gerade die diffuse „lingua franca“ erscheint indes als anschlussfähig genug, um die allgemeine Verankerung des Wettbewerbsprinzips im Feld der stationären Krankenversorgung abzusichern und zumindest auf der Ebene operationaler Governance dafür zu sorgen, dass die Akteure im fraglichen Spannungsfeld strukturbildend unterwegs sind und das Wettbewerbsdenken ,normalisieren“. Unsere Beobachtungen zeigen, wie sich dabei die Strukturation institutionellen Wandels vollzieht, wenngleich hier lediglich die kognitive Praxis als - allerdings konstitutives - Element dieses Strukturationsprozesses betrachtet wurde. Dieser Prozess vollzieht sich in einem Raum, in dem Uneindeutigkeit mit Interpretationen gefüllt und dann Ausgangsbasis materieller Handlungen wird. Beispielsweise ergibt sich aus diesem Strukturationsprozess, dass sich das Gros der Auseinandersetzungen auf die Organisation von Qualitätsbewerb bezieht. Ansonsten „leben“ die intermediären Kollektivakteure das Konkurrenzprinzip in ihrem praktischen Steuerungshandeln (beispielsweise bei der Feinsteuerung der DRGs); sie sind aber auch einbettend tätig, etwa indem sie (mehrheitlich) gegen Preiswettbewerb initiativ werden. In dieser Hinsicht werden also Konkurrenzbeziehungen in komplexer Weise „enaktiert“. Die Marktkultur als solche ist mithin im heutigen Krankenhauswesen ebenso allgegenwärtig wie hochgradig ambivalent, sie lässt vieles offen und ist - gerade deswegen - ultrastabil.

\section{Literatur}

Amelung, V.E. (2009): Managed Care - Neue Wege im Gesundheitsmanagement, in: Ders. / D. Deimel / W. Reuter / N. van Rooij / J.N. Weatherly (Hrsg.), Managed Care in Europe, Berlin, S. 3-30.

Augurzky, B. / R. Budde / S. Krolop / C.M. Schmidt / H. Schmidt / H. Schmitz / S. Terkatz / C. Schwierz (2009): Krankenhaus-Rating Report 2008. Qualität und Wirtschaftlichkeit, Essen: Rhein-Westfälisches Institut für Wirtschaftsforschung.

Augurzky, B. / S. Krolop / C.M. Schmidt (2010): Die wirtschaftliche Lage der Krankenhäuser, in: J. Klauber / M. Gareadts / J. Friedrich (Hrsg.), Krankenhausreport 2010. Krankenhausversorgung in der Krise?, Stuttgart, S. 13-24.

Ballast, T. (2011): Versorgung sinnvoll gestalten, in: Forum für Gesundheitspolitik 1-2, S. 4-7.

Bär, S. (2011): Das Krankenhaus zwischen ökonomischer und medizinischer Vernunft. Krankenhausmanager und ihre Konzepte, Wiesbaden.

Bauman, Z. (1991): Modernity and Ambivalence, Cambridge.

Beckert, J. (1997): Die Grenzen des Marktes. Die sozialen Grundlagen wirtschaftlicher Effizienz, Frankfurt / Main - New York.

Bode, I. (1998): Vermittlungsleistungen normativer Interessenorganisationen. Verbände im Gesundheitswesen als „enfants terribles“ mit Gemeinwohlbindung, in: Soziale Welt 49 / 2, S. 183-204.

Bode, I. (2005): Einbettung und Kontingenz. Wohlfahrtsmärkte und ihre Effekte im Spiegel der neueren Wirtschaftssoziologie, in: Zeitschrift für Soziologie 34 / 4, S. 250-269.

Bode, I. (2013): Die Infrastruktur des postindustriellen Wohlfahrtsstaats. Organisation - Wandel - Hintergründe, Wiesbaden.

Braun, B. / P. Buhr / S. Klinke / R. Rosenbrock (2010): Pauschalpatienten, Kurzlieger und Draufzahler Auswirkungen der DRGs auf Versorgungsqualität und Arbeitsbedingungen im Krankenhaus, Bern.

Bruckenberger, E. / S. Klaue / S. Schwintowski (2006): Krankenhausmärkte zwischen Regulierung und Wettbewerb, Berlin.

Döhler, M. / P. Manow (1995): Formierung und Wandel eines Politikfelds: Gesundheitspolitik von Blank zu Seehofer, MPIfG discussion paper 6, Köln: Max-Planck-Institut für Gesellschaftsforschung. 
Fley, B. (2006): Konkurrenz oder Distinktion? Wettbewerb und Marktkonzentration im deutschen Transportmarkt, in: M. Florian / F. Hillebrandt (Hrsg.), Pierre Bourdieu: Neue Perspektiven für die Soziologie der Wirtschaft, Wiesbaden, S. 169-195.

Fligstein, N. (2011): Die Architektur der Märkte, Wiesbaden.

Fuhse, J. / S. Mützel (2010): Relationale Soziologie. Zur kulturellen Wende der Netzwerkforschung, Wiesbaden.

Fürstenberg, T. et al. (2010): G-DRG Begleitforschung gemäß 17 b Absatz 8 KHG. Endbericht des ersten Forschungszyklus (2004 bis 2006), Berlin.

Giddens, A. (1992): Die Konstitution der Gesellschaft. Grundzüge einer Theorie der Strukturierung, Frankfurt / Main.

Grunow, D. (Hrsg.) (2006): Verwaltungshandeln in Politikfeldern, Wiesbaden.

Grzeszick, B. (2010): Wohlfahrt zwischen Staat und Markt. Korporatismus, Transparenz und Wettbewerb, Berlin.

Hessinger, P. (2009): Soziale Konstruktion von Märkten und integrierte Versorgung - Rationalitätskonflikte in der aktuellen Reorganisation des Gesundheitswesens, in: V.E. Amelung / J. Sydow / A. Windeler (Hrsg.), Vernetzung im Gesundheitswesen. Wettbewerb und Kooperation, Stuttgart, S. 51-74.

Heubel, F. / M. Kettner / A. Manzeschke (Hrsg.) (2010): Die Privatisierung von Krankenhäusern. Ethische Perspektiven, Wiesbaden, S. 165-194.

Hill, M. / P.L. Hupe (2009): Implementing Public Policy. An Introduction to the Study of Operational Governance, 2. Auflage, London.

Junge, M. (2000): Ambivalente Gesellschaftlichkeit. Die Modernisierung der Vergesellschaftung und die Ordnungen der Ambivalenzbewältigung, Opladen.

Leber, W. (2004): Qualitätssicherung in einem wettbewerblichen Umfeld, in: J. Klauber / B.-P. Robra / H. Schellschmidt (Hrsg.), Krankenhaus-Report 2004, S. 163-177.

Liebold, R. / R. Trinczek (2009): Experteninterview, in: S. Kühl / P. Strodtholz / A. Taffertshofer (Hrsg.), Handbuch Methoden der Organisationsforschung, Wiesbaden, S. 32-56.

Litwak, E. (1978): Drei alternative Bürokratiemodelle, in: R. Mayntz (Hrsg.), Bürokratische Organisationen, Köln - Berlin, S. 117-126.

Mayntz, R. (2009): Über Governance. Institutionen und Prozesse politischer Regulierung, Frankfurt / Main - New York.

Manzeschke, A. (2010): „Ohne Ansehen der Person“ - Zur ethischen Unterbestimmtheit der ökonomischen Theorie im Privatisierungsdiskurs, in: F. Heubell / M. Kettner / Ders. (Hrsg.), Die Privatisierung von Krankenhäusern. Ethische Perspektiven, Wiesbaden, S. 133-164.

Neckel, S. (2008): Flucht nach vorn. Die Erfolgskultur der Marktgesellschaft, Frankfurt / Main - New York.

Nullmeier, F. (2000): Politische Theorie des Sozialstaats, Frankfurt / Main - New York.

Nullmeier, F. (2007): Vermarktlichung des Sozialstaats?, in: D. Aufderheide / M. Dambrowski (Hrsg.), Markt- und Wettbewerb in der Sozialwirtschaft, Berlin.

Olten, Rainer (1998), Wettbewerbstheorie und Wettbewerbspolitik, München.

Paquet, R. (2011): Vertragswettbewerb in der GKV und die Rolle der Selektivverträge: Nutzen und Informationsbedarf aus der Patientenperspektive, Bonn: Friedrich Ebert Stiftung.

Pfaff, H. / F. Scheibler (2006): Versorgung und Versorgungsqualität im Krankenhaus: Standardisierung und Individualisierung, in: C. Wolf / C. Wendt (Hrsg.), Soziologie der Gesundheit. Sonderheft der Kölner Zeitschrift für Soziologie und Sozialpsychologie, Wiesbaden, S. 432-443.

Rau, F. / N. Roeder / P. Hensen (Hrsg.)(2009): Auswirkungen der DRG-Einführung in Deutschland. Standortbestimmung und Perspektiven, Stuttgart. 
Reay, T. / C.R.B. Hinings (2009): Managing the Rivalry of Competing Institutional Logics, in: Organization Studies 30 / 6, S. 629-652.

Scheibelhofer, E. (2008): Combining Narration-Based Interviews with Topical Interviews: Methodological Reflections on Research Practices, in: International Journal of Social Research Methodology 11 / 5, S. 403-416.

Simon, M. (2008): Personalabbau im Pflegedienst der Krankenhäuser. Hintergründe - Ursachen - Auswirkungen, Bern.

Slater, D. / F. Tonkiss (2001): Market Society: Markets and Modern Social Theory, Cambridge.

Thornton, P.H. / W. Ocasio / M. Lounsbury (2012): The Institutional Logics Perspective. A New Approach to Culture, Structure and Process, Oxford.

Weick, K.E. (1979): The Social Psychology of Organizing, 2. Auflage, Reading.

Vogd, W. (2011): Zur Soziologie der organisierten Krankenbehandlung, Weilerswist.

Prof. Dr. Ingo Bode

Dipl.-Soz. Markus Märker

Universität Kassel

FB 01 Humanwissenschaften

Arnold-Bode-Straße 10

34109 Kassel

ibode@uni-kassel.de maerker@uni-kassel.de 